\title{
BMJ Open Dose-response relationship between physical activity and mortality in people with non-communicable diseases: a study protocol for the systematic review and meta-analysis of cohort studies
}

\author{
Wolfgang Geidl, ${ }^{1}$ Sabrina Schlesinger, ${ }^{2}$ Eriselda Mino, ${ }^{1}$ Lorena Miranda, ${ }^{1}$ \\ Anna Ryan, ${ }^{1}$ Katja Bartsch, ${ }^{1}$ Lukas Janz, ${ }^{1}$ Klaus Pfeifer ${ }^{1}$
}

To cite: Geidl W, Schlesinger S, Mino E, et al. Dose-response relationship between physical activity and mortality in people with non-communicable diseases: a study protocol for the systematic review and meta-analysis of cohort studies. BMJ Open 2019;9:e028653. doi:10.1136/ bmjopen-2018-028653

- Prepublication history and additional material for this paper are available online. To view these files, please visit the journal online (http://dx.doi org/10.1136/bmjopen-2018028653).

Received 20 December 2018 Revised 06 August 2019 Accepted 16 August 2019

Check for updates

(c) Author(s) (or their employer(s)) 2019. Re-use permitted under CC BY-NC. No commercial re-use. See rights and permissions. Published by BMJ.

${ }^{1}$ Department of Sport Science and Sport, Friedrich-Alexander University Erlangen-Nürnberg, Erlangen, Germany

${ }^{2}$ Institute for Biometrics and Epidemiology, German Diabetes Center, Leibniz Center for

Diabetes Research at Heinrich Heine University, Düsseldorf, Germany

Correspondence to

Dr Wolfgang Geidl;

wolfgang.geidl@fau.de

\section{ABSTRACT}

Introduction This study protocol outlines our planned systematic review and dose-response meta-analysis of postdiagnosis physical activity and mortality in people with non-communicable diseases (NCDs).

Methods and analysis This study is based on the Preferred Reporting Items for Systematic Reviews and Meta-analysis for Protocols. A systematic literature search will be conducted in various databases-namely, PubMed, Scopus and Web of Science-by two researchers in order to identify prospective observational studies that investigate postdiagnosis physical activity or activityrelated energy expenditure and mortality in individuals with NCDs. The target population is adults ( $\geq 18$ years of age) with one of the following nine NCDs: low back pain, type 2 diabetes mellitus, osteoarthritis, depressive disorder, chronic obstructive pulmonary disease, breast cancer, lung cancer, stroke or ischaemic heart disease. We will focus on all-cause mortality as the primary outcome and investigate indication-specific mortality as the secondary outcome. For each study identified as a result of the literature search, we will conduct graphical doseresponse analyses of mortality as a function of activityrelated energy consumption. If more than two studies are available for one disease, we will perform linear and non-linear dose-response meta-analyses for said disease using random-effects models. We will investigate the heterogeneity of the studies and publication bias. To assess the risk of bias and the quality of the included studies, we will use the Risk Of Bias In Non-randomised Studies - of Interventions tool, which is a Cochrane tool. Ethics and dissemination This systematic review will be conducted in compliance with ethical precepts. As the systematic review is based on published studies, approval from an ethics committee is not required. The systematic review and meta-analysis will be published in a peerreviewed journal.

PROSPERO registration number CRD42018103357

\section{INTRODUCTION}

The World Health Organization (WHO) recommends at least $150 \mathrm{~min}$ of moderate-intensity physical activity or $75 \mathrm{~min}$ of
Strengths and limitations of this study

- Our systematic review will be conducted and reported in accordance with the reporting guidelines provided in the Preferred Reporting Items for Systematic Reviews and Meta-analysis for Protocols statement and the reporting guidelines of the Metaanalysis Of Observational Studies in Epidemiology.

- The scope of our systematic search is wide-reaching, as it includes nine non-communicable diseases and three extensive medical databases.

- The study uses the novel Risk Of Bias In Nonrandomised Studies - of Interventions tool.

- However, the observational cohort studies do not provide a conclusive answer regarding the causality between physical activity and mortality.

vigorous-intensity physical activity per week to enhance health and reduce mortality. ${ }^{1}$ For additional benefits, adults should increase their moderate-intensity physical activity to $300 \mathrm{~min}$ or engage in $150 \mathrm{~min}$ of vigorous-intensity physical activity per week. These recommendations apply to both healthy adults and adults with non-communicable diseases (NCDs) (eg, ischaemic heart disease, breast cancer, chronic pulmonary disease). However, if one considers the scientific evidence for physical activity and mortality on which the recommendations are based, an extensive disparity becomes apparent between healthy populations and those with a pre-existing NCD.

The data for healthy adults are comprehensive and unambiguous. Numerous large cohort studies have consistently demonstrated an inverse relationship between physical activity and mortality. ${ }^{2}$ Arem et al. $^{3}$ pooled data from six cohort studies of 661137 persons. Compared with individuals who 
reported having no leisure-time physical activity, premature death decreased with increased physical activity levels: 7.5 metabolic equivalent tasks (MET) hours/week (HR $=0.80 ; 95 \%$ CI 0.78 to 0.82 ); $7.5-15$ MET hours/week (HR=0.69; 0.67 to 0.70); and 15-22.5 MET hours/week $(\mathrm{HR}=0.63 ; 0.62$ to 0.65$)$. These findings are consistent with the meta-analysis conducted by Samitz et al. ${ }^{4}$ This analysis comprised 80 studies with a total of 1338143 persons. Compared with the lowest activity group, the risk of premature death was remarkably reduced in the highest activity group ( $\mathrm{HR}=0.65 ; 95 \% \mathrm{CI} 0.60$ to 0.71 ). Furthermore, each 1 hour increment of moderate-intensity activity per week resulted in a lowered risk ratio (RR) of 0.96 (95\% CI 0.93 to 0.98 ).

Accordingly, the updated physical activity guidelines from the US Department of Health and Human Services ${ }^{5}$ include a clear dose-response relationship between the volume of physical activity and the mortality rates of healthy adults. The shape of the dose-response curve is not linear but regressive, thus meaning that the greatest difference in mortality rates occurs among inactive and minimally active individuals. It is clear that benefits can be gained with any amount of physical activity. For healthy individuals, current scientific research is sceptical of a minimum dose of physical activity to ensure lifetime extension. Following the minimum recommendations, physical activity is equivalent to energy expenditure of 8.25 MET hours/week. At this level of physical activity, about $70 \%$ of the benefits in relation to mortality rates are reached. ${ }^{6}$ Higher volumes of physical activity mean that the dose-response curve flattens out. However, roughly five times this dose is also associated with more risk reductions and no adverse effects.

For individuals with distinct NCDs, the scientific data on the dose-response relationship between physical activity and mortality are considerably weaker. For cancer, the meta-analysis by $\mathrm{Li}$ et $a l^{7}{ }^{7}$ suggests that postdiagnosis physical activity levels may result in similar risk reductions in mortality. Moore et al. ${ }^{8}$ pooled data from six cohort studies that comprised 654827 individuals and adjusted their analysis for several confounders, including pre-existing NCDs. In contrast to $\mathrm{Li}$ et al., ${ }^{7}$ they conclude that the longevity effects of physical activity vary according to the pre-existing NCD. The current evidence from the US Physical Activity Guidelines Advisory Committee ${ }^{6}$ reports a general relationship between higher postdiagnosis physical activity and lower mortality rates in five NCDs (breast cancer, colorectal cancer, prostate cancer, cardiovascular condition of hypertension and type 2 diabetes). However, this report did not demonstrate the dose-response relationships due to the limited information it had regarding the NCDs that were worked on. In addition, the report does not include all NCDs with high levels of morbidity and mortality in Western countries. In Germany, the following NCDs are in the top 10 NCDs with the highest burden of disease: ischaemic heart disease, low back pain, lung cancer, breast cancer, stroke, chronic obstructive pulmonary disease (COPD), major depressive disorder and diabetes. ${ }^{9}$ The high disease burden of these NCDs refers to the loss of life due to premature death and years spent living with a disability as a result of the disease. For some NCDs, such as low back pain or major depressive disorder, the high burden is mainly caused by a loss of healthy years. However, the data from Plass et al. ${ }^{9}$ also show at least a small influence on mortality rates. Overall, it is unclear whether physical activity positively affects mortality rates in individuals with NCDs in the same way that physical activity affects the mortality rates of healthy individuals. Thus, it is clear that the dose-response relationship between physical activity and mortality in adults with a NCD is not well defined at present.

\section{Objectives}

This study protocol aims to describe the planned systematic review and dose-response meta-analysis of physical activity and mortality in adults with NCDs. The planned study aims to define the dose-response relationship between postdiagnosis physical activity and mortality rates for nine NCDs with a high global burden of disease, ${ }^{10}$ especially in Germany. ${ }^{9}$ The nine NCDs are: low back pain, type 2 diabetes mellitus, osteoarthritis, depressive disorder, COPD, breast cancer, lung cancer, stroke and ischaemic heart disease. Our results may inform updates on national physical activity recommendations for individuals with NCDs. ${ }^{56}$ The planned dose-response analyses may help specify the recommended amount of physical activity and define a minimum, optimum and maximum dose of physical activity for individuals with NCD.

\section{METHODS}

This protocol adheres to the Preferred Reporting Items for Systematic Reviews and Meta-analysis for Protocols (online supplementary file 1 ). ${ }^{11}$ Our systematic review will be conducted and reported in accordance with the reporting guidelines provided in the Preferred Reporting Items for Systematic Reviews and Meta-Analysis statement and the Meta-analysis Of Observational Studies in Epidemiology reporting guidelines. ${ }^{12}{ }^{13}$ Additionally, the Methodological Expectations of Cochrane Intervention Reviews and the Cochrane Handbook of Systematic Reviews in Interventions will be consulted to ensure methodological quality. ${ }^{1415}$

In view of the recommendations that endorse the preregistration of systematic reviews, our protocol was registered with the International Prospective Register of Systematic Reviews on 5 September 2018 (available online at https://www.crd.york.ac.uk/prospero/display_ record.php?RecordID=103357).

\section{Eligibility criteria}

This study will only include research published in the English language. There are no time restrictions in relation to the year of publication. We will include studies that investigate the association between postdiagnosis physical activity levels and mortality among adults with NCD and report on the effect estimates, including the 
HRs, relative risks, ORs or absolute mortality rates. ${ }^{12}{ }^{16}$ For this study, postdiagnosis physical activity will be defined as any form of physical activity, such as leisure-time, occupational, transport-related, exercise and any physical activity-related energy expenditure measured after diagnosis. Physical activity can be measured using subjective methods (eg, questionnaires) or objective methods (eg, accelerometry). Physical activity-related energy expenditure can be measured using any kind of objective method (eg, doubly labelled water).

Studies will be excluded if they: (1) clearly deal with another topic; (2) include only the total population without information for subgroups with NCDs at the baseline; (3) focus on prevention only (ie, when they include individuals at risk of developing one of the nine diseases); (4) report insufficient data (ie, less than three different physical activity levels in MET hours per week) to calculate the dose-response relationship; (5) are duplicate studies that are based on a data set that has already been taken into account.

\section{Participants}

The participants for the study will be comprised of those who are $\geq 18$ years of age with at least one of the following nine NCDs at the baseline: osteoarthritis, low back pain, depressive disorder, ischaemic heart disease, type 2 diabetes mellitus, stroke, COPD, lung cancer or breast cancer. The disease can either be confirmed by a physician or determined by self-reporting. Studies that have children, adolescents and pregnant women as the participants will be excluded, as will studies that focus on animal and cell cultures.

\section{Outcomes}

The outcomes will be studies that assessed all-cause mortality as the primary endpoint or any indication-specific mortality as the primary or secondary endpoint.

\section{Study design}

Prospective observational studies, including cohort, nested case-control, case-cohort studies and follow-up studies of randomised controlled studies published in a peer-reviewed journal will be included. We will exclude cross-sectional, case only or casecontrol studies, conference abstracts, comments, letters and reviews.

\section{Information sources}

Two researchers will search the following electronic databases: MEDLINE (PubMed), Scopus and the Web of Science Core Collection (Web of Science). All years will be covered. The reference list from the systematic reviews and meta-analyses will be manually searched to locate further results. Additionally, one researcher will use the Google Scholar forward citation search for all eligible articles identified via the database search.

\section{Search strategy}

The search strategy was developed with the support of a specialist from the University Library. The search is structured according to three main categories of the population, intervention, comparison, outcome (PICO) concept. The population is people with one of the nine NCDs; the intervention is the physical activity; the outcome is mortality; and control, as the fourth category of PICO, does not play a role in the cohort studies we sought out. ${ }^{17}$ We defined the search terms for the three PICO categories; these terms included keywords and related synonyms, abbreviations, spelling variations and controlled vocabulary, each separated by Boolean operator OR. The search terms for the three PICO categories will be combined with Boolean operator AND. The search will be restricted to the search fields of the title and the abstract. Independent searches will be conducted for the nine NCDs under consideration. The search is adapted to the special features of the three databases (eg, the use of medical subject heading terms in PubMed). It should be noted that the search is not filtered for observational studies, as reference lists of systematic reviews and meta-analyses are eligible for additional manual searching. The concrete search terms used can be found in online supplementary file 2 .

\section{Data management}

The search results will be imported to the reference management and knowledge organisation software, Citavi V.5. We will use separate project folders for each of the nine NCDs. These folders will be organised hierarchically in categories, based on the various inclusion and exclusion filters.

\section{Selection of eligible studies}

First, one researcher will screen each article's title and abstract against the eligibility criteria to identify all relevant studies. Then, a second researcher will perform the same screening task to ensure that no studies were overlooked or incorrectly included. This procedure will have a positive effect on the accuracy and reliability of the screening process. ${ }^{18}$ Moreover, increasing the number of contributors in this critical point of the systematic review enables the improved timeliness and efficiency of the process. ${ }^{19}$ If the screening process of the title and abstract does not lead to a clear result, the article will be retrieved for full-text screening.

\section{Data extraction}

Data of the full texts will be independently extracted by two reviewers using an Excel table (online supplementary file 3). This table has been pilot-tested with a number of eligible articles from four reviewers (AR, EM, LM, WG). The ensuing discussion secured a mutual understanding of the variables, the standardisation of the Excel data mask and a uniform system of data extraction. The results of the double data extraction will be checked for consistency. Any disagreements will be openly discussed by the three reviewers. Multiple publications with the same or very similar content will only be considered once; 
duplicates with smaller sample sizes and shorter follow-up durations will be excluded.

\section{Data items}

The information for extraction includes basic details such as the name of the first author, year of publication, study name, design, country where research was undertaken, age and sex of participants and mean follow-up time. Additionally, we will retrieve data regarding the total sample, total all-cause death cases, the number of participants in each physical activity category, death cases per the corresponding category, diagnosis and mortality data ascertainment, exposure to physical activity (eg, MET hours/week, $\min /$ day) and any corresponding categories. Finally, RR with $95 \%$ CIs will be extracted from fully adjusted models for every physical activity exposure category, as well as for doseresponse data, when available.

\section{Outcomes}

The primary outcome of this review will be all-cause mortality, defined as the number of deaths over the entire period of follow-up, regardless of the underlying cause of death. As previously discussed, overall mortality is one of the main investigated types of death attributable to a lack of physical activity in persons affected by NCD. The relationship between physical activity and longevity is complex, ${ }^{20}$ and during a certain timeframe, death can be caused or affected by multiple factors. Hence, disease-specific standardised death rates can exclude many cases that can blur the identification of a possible causal relation. If all-cause mortality rates are not reported, disease-specific mortality rates will be considered. Thus, the secondary outcomes include indication-specific mortalities such as breast cancer mortality.

\section{Risk of bias assessment}

Assessment of bias across the included studies is very important, as the results can affect the variability among single studies and consequently, the meta-analysis. ${ }^{21} \mathrm{We}$ will use the Cochrane Risk Of Bias In Non-randomised Studies - of Interventions (ROBINS-I) for assessing bias. ${ }^{22}$ This tool pays particular attention to the internal validity of a study by comparing it to a hypothetical randomised controlled trial (RCT). The external validity of the study is not considered in this tool, and any generalisability, applicability or ethical issues will not affect our judgement.

ROBINS-I is a domain-based method of assessing the risk of bias. Seven domains are included in total. Confounding factors and selection bias have always been a matter of importance in observational study designs, and both of these elements constitute two essential domains of ROBINS-I. ${ }^{23}$ The additional domains of ROBINS-I include the classification of interventions, deviations from intended interventions, missing data, measurement of outcomes and selection of the reported results. ${ }^{24}$ Through ROBINS-I, systematic appraisal is conducted in three phases.

\section{Phase I}

The protocol stage focuses on any general forethoughts to be considered prior to appraising each study. This stage specifies the review question, identifies the relevant confounding domains for the included studies and notes possible cointerventions (exposures) that have an impact on study outcomes.

\section{Phase II}

The second stage is concerned with hypothesising a RCT and elaborating on the confounders and cointerventions for each study.

\section{Phase III}

The final stage focuses on the actual appraisal in the seven domains that expose the study to the risk of bias. This instrument contains five options to answer the signalling questions-namely, yes, probably yes, no, probably not and no information. In the same manner, the domain-specific judgments are based on five categories-namely, low, moderate, serious, critical risk and no information.

Each study will be independently rated by two reviewers, and any disagreement will be first noted and then followed by a discussion and consultation with a third group member. The final assessment will result in a table that includes all of the studies along with the domain-specific and overall conclusions reached by the reviewers.

\section{Meta-biases assessment}

We are aware of the implication of meta-biases (eg, sampling, selection and data extraction bias) for the internal validity of this study. ${ }^{25}$ To minimise meta-biases, the entire process will follow the suggestions of the above guidelines. Retrieval bias will be minimised with a comprehensive and representative search strategy. If the number of included studies permits this, publication bias will be assessed via funnel plots. ${ }^{26}$ To minimise selection bias, inclusion criteria were selected on the basis of a comprehensive discussion. Furthermore, we will employ a double-check screening method against a clearly defined and specific criterion for eligibility. To address extractor biases, we will use a double-check approach of data extraction, which has been proven to improve the extraction process. ${ }^{27} 28$ This review is limited to peer-reviewed published literature. A supplementary search for unpublished studies and literature will not occur, thus meaning that, to a certain extent, this review is susceptible to grey literature bias. ${ }^{29}$

\section{Synthesis of results}

First, following the methodological approach of Warburton and Bredin, ${ }^{2}$ for each identified study, we will conduct graphical dose-response analyses of mortality as a function of activity-related energy consumption. The data regarding the dose of physical activity will be converted into a single unit (ie, MET hours/week). Only studies that investigate exposure to at least three different levels of physical activity will be included in the dose-response analysis. If the physical activity categories 
are defined without assigning a specific value for energy expenditure, we will assume the corresponding absolute intensities to be 1.5-3.0 MET for a low level of physical activity; 3-6 MET for moderate physical activity; and $\geq 6$ MET for a high level of physical activity. ${ }^{30} 31$ When studies report the duration of different physical activities (eg, 30 min of walking, running or cycling), we will calculate the energy expenditure based on the compendium of physical activities. ${ }^{30}$

Second, for each of the nine NCDs, summary RRs with 95\% CIs will be calculated when two or more studies of the same exposure and outcome are available. We will apply random effects meta-analysis, as described by DerSimonian and Laird. ${ }^{32}$ If a study reports on separate risk estimates for subgroups (eg, men and women), we will pool the data using a fixed-effect model and include the combined estimate in the overall meta-analysis.

Third, indication-specific linear dose-response meta-analyses will be conducted using the method described by Greenland and Longnecker. ${ }^{33}$ In addition, we will investigate the shape of the association by conducting non-linear dose-response meta-analysis, as described by Orsini et al. ${ }^{34}$ For this method, the following data for at least three exposure categories are required: (1) the quantified exposure value (MET hours/weeks); (2) the effect estimate with the corresponding $95 \% \mathrm{CI}$; and (3) the number of cases and person-years. If the information regarding the distribution of cases, personyears or non-cases is missing, data will be estimated as previously described. ${ }^{35} 36$ The mean amount of exposure between two endpoints for each physical activity category will be calculated. ${ }^{2}$ When the lowest or highest category is open-ended (eg, <3), we will multiply the value by 1.25 .

Heterogeneity will be described by calculating Tau ${ }^{2}$ to assess the between-study variance and calculating the $\mathrm{I}^{2}$ statistic to investigate the variability of the observed effects in the meta-analyses. ${ }^{37}$ Possible sources of heterogeneity across the studies will be explored by conducting subgroup analyses and meta-regressions by accounting for various factors (eg, sex, age, geographic location of the studies, follow-up time, assessment of physical activity, risk of bias of the studies). The small-studies effect (eg, publication bias) will be investigated by conducting visual inspections of the funnel plots and applying Egger's test, at which $\mathrm{p}<0.1$ indicates potential publication bias. ${ }^{38}$ Data analyses will be performed using the statistical software Stata V.15. All tests will be two-sided, with statistical significance defined as $\mathrm{p}<0.05$.

\section{Patient and public involvement}

As the systematic review will be based on published studies, patient or public involvement is not applicable.

\section{Limitations}

Some potential limitations are to be expected. First, prospective observational cohort studies fail to provide conclusive evidence of a causal relationship between physical activity and mortality. ${ }^{20}{ }^{39-41}$ Consequently, our review of cohort studies does not provide a conclusive answer as to whether the reported relationships between physical activity and mortality are actually causal or only correlative. According to Hill, ${ }^{42}$ however, confidence in a causal relationship increases when (1) a clear dose-response curve, (2) a strong association or a high effect size and (3) consistency of results in different studies are given. These three factors will be examined in our systematic review. Thus, this work can contribute to estimations of the likelihood of the causal influence of physical activity on mortality rates. Second, we will only include studies published in English. Studies published in other languages and grey, unpublished literature will not be included. Third, the wide range of tools available to measure physical activity in terms of their psychometric properties and the domains that they assess may present another challenge. This variability in measurement instruments may present difficulties in generating one single energy metric unit of physical activity, thus questioning the inclusion of all the eligible studies in the doseresponse analysis. However, we will consider any form of physical activity by representing it in associated energy consumption units, and we will not consider potential differences between different intensities (ie, light vs moderate vs vigorous) or between physical activity in different contexts (eg, leisure time physical activity vs occupational physical activity). Fourth, this study will only consider activity behaviour, not sedentary behaviour, even if there is a clear interaction between physical activity and sedentary behaviour with regard to mortality in healthy individuals. $^{43}$

Contributors WG had the initial idea for this review; he is the guarantor of the study. WG, EM, SS, LM, AR and KP designed the study, including the development of the selection criteria, the risk of bias assessment strategy, the search strategy and the data extraction strategy. KB and LJ will monitor the screening process. $A R, E M$ and LM will retrieve the data from the studies qualified for inclusion. SS will conduct the meta-analysis. EM, WG and SS prepared the draft of this study protocol. All authors contributed substantially to the drafting of the paper and its revisions. All authors have read and approved the final manuscript.

Funding The authors have not declared a specific grant for this research from any funding agency in the public, commercial or not-for-profit sectors.

Competing interests None declared.

Patient consent for publication Not required.

Provenance and peer review Not commissioned; externally peer reviewed.

Open access This is an open access article distributed in accordance with the Creative Commons Attribution Non Commercial (CC BY-NC 4.0) license, which permits others to distribute, remix, adapt, build upon this work non-commercially, and license their derivative works on different terms, provided the original work is properly cited, appropriate credit is given, any changes made indicated, and the use is non-commercial. See: http://creativecommons.org/licenses/by-nc/4.0/.

\section{REFERENCES}

1. World Health Organization. Global recommendations on physical activity for health. Geneva, Switzerland: World Health Organization, 2010.

2. Warburton D, Bredin S. Health benefits of physical activity: a systematic review of current systematic reviews. Curr Opin Cardiol 2017;32:541-56.

3. Arem $\mathrm{H}$, Moore SC, Patel A, et al. Leisure time physical activity and mortality: a detailed pooled analysis of the dose-response relationship. JAMA Intern Med 2015;175:959-67. 
4. Samitz G, Egger M, Zwahlen M. Domains of physical activity and allcause mortality: systematic review and dose-response meta-analysis of cohort studies. Int J Epidemiol 2011;40:1382-400.

5. U.S. Department of Health and Human Services. Physical activity guidelines for Americans. 2nd edition. Washington, DC: U.S.: Department of Health and Human Services, 2018.

6. 2018 Physical Activity Advisory Committee. 2018 physical activity guidelines Advisory Committee scientific report. Washington, DC: U.S.: Department of Health and Human Services, 2018.

7. Li T, Wei S, Shi Y, et al. The dose-response effect of physical activity on cancer mortality: findings from 71 prospective cohort studies. $\mathrm{Br} \mathrm{J}$ Sports Med 2016;50:339-45.

8. Moore SC, Patel AV, Matthews CE, et al. Leisure time physical activity of moderate to vigorous intensity and mortality: a large pooled cohort analysis. PLoS Med 2012;9:e1001335.

9. Plass D, Vos T, Hornberg C, et al. Trends in disease burden in Germany: results, implications and limitations of the global burden of disease study. Dtsch Arztebl Int 2014;111:629-38.

10. Murray CJL, Vos T, Lozano R, et al. Disability-adjusted life years (DALYs) for 291 diseases and injuries in 21 regions, 1990-2010: a systematic analysis for the global burden of disease study 2010. The Lancet 2012;380:2197-223.

11. Moher D, Shamseer $L$, Clarke $M$, et al. Preferred reporting items for systematic review and meta-analysis protocols (PRISMA-P) 2015 statement. Syst Rev 2015;4:1.

12. Moher D, Liberati A, Tetzlaff J, et al. Preferred reporting items for systematic reviews and meta-analyses: the PRISMA statement. BMJ 2009;339. 10.1136/bmj.b2535.10.1371/journal.pmed.1000097.

13. Stroup DF, Berlin JA, Morton SC, et al. Meta-Analysis of observational studies in epidemiology: a proposal for reporting. meta-analysis of observational studies in epidemiology (moose) group. JAMA 2000;283:2008-12.

14. Higgins JPT, Lasserson T, Chandler J, et al. Methodological expectations of Cochrane intervention reviews. London: Cochrane, 2016.

15. Higgins JPT, Green S, eds. Cochrane handbook for systematic reviews of interventions cochrane book series. The Cochrane Collaboration, 2011.

16. Richardson WS, Wilson MC, Nishikawa J, et al. The well-built clinical question: a key to evidence-based decisions. ACP J Club 1995;123:A12-13.

17. Schardt C, Adams MB, Owens T, et al. Utilization of the PICO framework to improve searching PubMed for clinical questions. BMC Med Inform Decis Mak 2007;7:16.

18. Edwards P, Clarke M, DiGuiseppi C, et al. Identification of randomized controlled trials in systematic reviews: accuracy and reliability of screening records. Stat Med 2002;21:1635-40.

19. $\mathrm{Ng} \mathrm{L}$, Pitt V, Huckvale K, et al. Title and Abstract screening and evaluation in systematic reviews (TASER): a pilot randomised controlled trial of title and Abstract screening by medical students. Syst Rev 2014;3:121.

20. Kujala UM. Is physical activity a cause of longevity? it is not as straightforward as some would believe. A critical analysis. $\mathrm{Br} \mathrm{J}$ Sports Med 2018;52:914-8.

21. Higgins JP, Ramsay C, Reeves BC, et al. Issues relating to study design and risk of bias when including non-randomized studies in systematic reviews on the effects of interventions. Res. Syn. Meth. 2013;4:12-25.

22. Sterne JAC, Hernán MA, Reeves BC, et al. ROBINS-I: a tool for assessing risk of bias in non-randomised studies of interventions. $B M J ; 355$.
23. Garcia-Doval I, van Zuuren EJ, Bath-Hextall F, et al. Systematic reviews: let's keep them trustworthy. Br J Dermatol 2017;177:888-9.

24. Sterne JA, Higgins JPT, Elbers RG, et al. Risk of bias in Nonrandomized studies of interventions (ROBINS-I): detailed guidance, updated 20 October 2016, 2016.

25. Felson DT. Bias in meta-analytic research. $J$ Clin Epidemiol 1992;45:885-92.

26. Sterne JAC, Harbord RM. Funnel plots in meta-analysis. Stata J 2004;4:127-41.

27. Mathes T, Klaßen P, Pieper D. Frequency of data extraction errors and methods to increase data extraction quality: a methodological review. BMC Med Res Methodol 2017;17:152.

28. Buscemi N, Hartling L, Vandermeer B, et al. Single data extraction generated more errors than double data extraction in systematic reviews. J Clin Epidemiol 2006;59:697-703.

29. Song F, Parekh, S, Hooper L, et al. Dissemination and publication of research findings: an updated review of related biases. Health Technol Assess 2010;14:1-193.

30. Ainsworth BE, Haskell WL, Herrmann SD, et al. 2011 compendium of physical activities: a second update of codes and Met values. Med Sci Sports Exerc 2011;43:1575-81.

31. Haskell WL, Lee I, Pate RR, et al. Physical activity and public health: updated recommendation for adults from the American College of sports medicine and the American heart association. Med Sci Sports Exerc 2007;39:1423-34.

32. DerSimonian R, Laird N. Meta-Analysis in clinical trials revisited. Contemp Clin Trials 2015;45:139-45.

33. Greenland S, Longnecker MP. Methods for trend estimation from summarized dose-response data, with applications to meta-analysis. Am J Epidemiol 1992;135:1301-9.

34. Orsini N, Li R, Wolk A, et al. Meta-Analysis for linear and nonlinear dose-response relations: examples, an evaluation of approximations, and software. Am J Epidemiol 2012;175:66-73.

35. Chene G, Thompson SG. Methods for summarizing the risk associations of quantitative variables in epidemiologic studies in a consistent form. Am J Epidemiol 1996;144:610-21.

36. Aune D, Greenwood DC, Chan DSM, et al. Body mass index, abdominal fatness and pancreatic cancer risk: a systematic review and non-linear dose-response meta-analysis of prospective studies. Ann Oncol 2012;23:843-52.

37. Borenstein M, Higgins JPT, Hedges LV, et al. Basics of meta-analysis: 2 is not an absolute measure of heterogeneity. Res Synth Methods 2017;8:5-18

38. Egger M, Smith GD, Schneider M, et al. Bias in meta-analysis detected by a simple, graphical test. BMJ 1997;315:629-34.

39. Shiroma EJ, Lee I-M. Can we proceed with physical activity recommendations if (almost) NO clinical trial data exist on mortality? Br J Sports Med 2018;52:888-9.

40. Wade KH, Richmond RC, Davey Smith G. Physical activity and longevity: how to move closer to causal inference. Br J Sports Med 2018;52:890-1.

41. O'Donovan G, Blazevich AJ, Boreham C, et al. The ABC of physical activity for health: a consensus statement from the British association of sport and exercise sciences. J Sports Sci 2010;28:573-91.

42. Hill $A B$. The environment and disease: association or causation? Proc R Soc Med 1965;58:295-300.

43. Ekelund U, Steene-Johannessen J, Brown WJ, et al. Does physical activity attenuate, or even eliminate, the detrimental association of sitting time with mortality? A harmonised meta-analysis of data from more than 1 million men and women. The Lancet 2016;388:1302-10. 